\title{
A DIFFERENT FIRST COURSE IN ELECTRICAL ENGINEERING
}

\author{
Don H. Johnson and J. D. Wise, Jr. \\ Department of Electrical and Computer Engineering \\ Rice University, MS 366 \\ Houston, Texas 77005-1892 \\ dhj@rice.edu, jdw@rice.edu
}

\begin{abstract}
Traditional introductory courses in electrical engineering are typically circuit theory courses, which may include both analog and digital hardware and possibly software. Recent alternatives have focused on how to teach (using discrete-time signals rather than analog) than on what to teach. We developed a top-down course sequence that uses as its underlying principle the transmission and manipulation of information. Students are given a broad perspective of both analog and digital approaches, with the goals of helping students appreciate electrical and computer engineering and framing a context for advanced courses. Laboratories stress construction of analog systems and analysis with signal processing tools.
\end{abstract}

\section{INTRODUCTION}

To set the context for developing our course, a little background. Rice University has a relatively small engineering school. Out of a total undergraduate population of about 2,500 , roughly $30 \%$ are engineering students. The Electrical and Computer Engineering Department is the largest in the engineering school, graduating 4050 bachelor's students each year. Our engineering graduates are well recruited, both by industry and graduate schools. The educational mission of Rice's engineering program, which crystallized over the past year or so as part of the engineering school's strategic planning process, is to educate technological leaders through coursework and interdisciplinary projects that stress fundamentals and what we term the engineering context: the milieu within which engineers must practice.

This goal led us to rethink what the first course in electrical engineering should be. Changing introductory courses has occurred in many schools, but our informal survey revealed that they tend to stress how to present concepts rather than addressing what concepts should be taught. Furthermore, if changing courses meant repackaging the same material, with upper-level courses going on as before, nothing has really been accomplished. While the adage "If it ain't broke, don't fix it" may have applied at one time, we felt that a more forward looking view was needed and that the curriculum needed constant attention rather than periodic review. At least at Rice, we continued to use circuit theory as the first electrical engineering course even as the deparment changed its name to Electrical and Computer Engineering over fifteen years ago.

To paraphrase thoughts presented by Dr. Andrew Viterbi during his Brice Lecture given at Rice about a year ago, the fundamental technology needed by undergraduates should be taught early and often. Certainly before 1950, that technology was circuit the- ory, as all implementations consisted of analog circuits. Once the inventions of the digital computer, the transistor, and Shannon's information theory occurred between 1946 and 1948, implementation choices and what was designed widened dramatically. Thus, no single core subject can be identified; instead, breadth is suggested. This line of thought lead us to rethink not only what technology should be thought first, but also to take a breadth-first rather that depth-first approach to education.

\section{COURSE DESIGN}

Prior to this year, our introductory electrical engineering course was a single semester course in circuit theory, normally taken in the sophomore year. Early in 1997 we decided to implement our idea of a breadth-first introductory course, focusing on the technology and engineering science that define modem electrical engineering. We quickly found that our room to expand breadth was constrained by the need to provide prerequisite basics for succeeding courses: While we wanted the new course to instill an appreciation for the big picture, we still needed to implant basic knowledge and skills. Although we could eliminate or postpone much of the "classical" circuit theory we had been teaching, considering the basics we needed to keep and the breadth we wanted to add, we were looking at a very "dense" course. The obvious solution was to add a second semester.

Once this decision was made (spring of 1997), we were ready to begin. Our goal was a new two-course introductory sequence that presents virtually all of the electrical engineering topics students will leam in detail as they progress during their major. The unifying theme of our courses is information theory: the representation, manipulation, control, transmission, and reception of information by electrical means. Both digital and analog approaches are included, with the importance of new devices brought forward as examples of what the students (not us) will face as design alternatives. We focus from the beginning not on technique but on goal: What is the engineer trying to do and what are some altematives to problem solution. Our intent is not to develop a complete framework for evaluating technologies within the course, but to inspire students, begin a systems-level, top-down design approach, and start them thinking about what their technological contributions might be. Design is not emphasized. We felt that to cover broadly information and control technology meant that they would be illprepared to perform well in realistic design situations. Rather, we stress alternatives from a broad view, as would occur in the beginning stages of top-down design. Open-ended situations occur throughout in the form of problem sets that are not drill-oriented. 
We also emphasize working in groups, both on problem sets and in the laboratory. In summary, we try to set the stage for succeeding courses so that students implicitly know the application of advanced material presented in upper-level courses.

\section{COURSE STRUCTURE}

When the dust had settled, we had apportioned the material for the two courses. The first course would deal with circuits, signals, and communication systems, primarily from a frequency domain viewpoint. The second course would take a time domain approach and would concentrate on control, electronics, and digital systems. Each course would introduce and employ basic concepts as required. The course URLs are www-ece.rice.edu/-elec241 and www-ece.rice.edu/ elec242; the topics (in sequence) covered in the courses are:

\section{Fundamentals of Electrical Engineering I}

- Introduction to signals and systems. Fundamental model of communication.

- Analog signals as voltages and currents. Circuit element voltage-current relations in time and frequency (impedance) domains. Transfer functions and the notion of filtering, with implementation by operational amplifiers.

- Frequency-domain analysis of signals: Fourier series, Fourier transforms, Parseval's Theorem, and spectrograms. Fundamental model of speech.

- Analog communication: amplitude modulation transmitted through additive white noise channels.

- Digital signal analysis: Sampling theorem, A/D conversion, difference equations, DTFT, DFT, and FFT. Analog and digital alternatives to filtering.

- Digital communication: Source coding theorem, Huffman codes, compression (lossless and lossy), signal sets, and matched filter receivers. Noisy channel coding theorem and error correcting codes. Comparison of analog and digital (PCM) communication.

- Ethernet as an example computer network.

\section{Fundamentals of Electrical Engineering II}

- Transducers as signal sources. Review of circuit theory, with an emphasis on the time-domain. Solution of dynamic equations. Laplace transform methods; notions of poles and zeros.

- Semiconductor devices: diodes, optoelectronic devices, operational amplifiers, and transistors.

- Digital circuits: gates and timing circuits.

- Feedback control, especially in the context of electromechanical systems.

Students who have taken the first course leave as familiar with the frequency domain as previous students were with Ohm's Law.

In the laboratory portion of the course, we revamped not just the context, but also the style. Previously the lab was a somewhat regimented experience. Students would arrive at the beginning of the scheduled session, check out a set of equipment (scope, function generator, multimeter, power supply, and breadboard), and carry it to a bench. Parts were drawn as needed from a set of cabinets containing standard components. At the end of the session, circuits would be disassembled and parts and equipment returned to the storeroom.

Our first change was to permanently set up the equipment on the benches to reduce setup overhead and allow flexibility in access to laboratory facilities. We gave each lab group (two students) their own breadboard and a kit of electric and electronic parts, including both those known to be required for course laboratories and an assortment of additional components for adjustments and experimentation. In addition, each student is given a set of basic tools that will be useful in both this and all succeeding hardware courses. Armed with tools and parts, students are encouraged to work outside of laboratory hours on any project they wish, using laboratory equipment if they wish.

In addition to the analog instruments in each lab station, we added PCs running Netscape, LabView from National Instruments, and Matlab. This addition significantly improved both the capabilities and the efficiency of the lab. The lab manual is kept on line as a web document (profusely illustrated in the early labs as students are introduced to components and instrumentation). Reference data such as the color code and component data sheets are also available.

We use LabView both for its "intended" purpose of building "virtual instruments" (spectrum analyzer, true RMS voltmeter, frequency response measurement, distortion analyzer) and to perform real-time digital signal processing (filtering, quantization, control law implementation). Matlab is used for more involved, "batch" signal processing, such as filter design and spectrogram display. Students were not asked to program in either environment, and used LabView as another instrument and Matlab for its computational power.

In the first course, one of the main themes is signals, and the lab seeks to reinforce this by instilling a natural, intuitive feeling for signals as real objects rather than simply an abstract concept. It does this by concentrating on signals that can be heard. The very first lab introduces students to "the sound of signals." Subsequent labs continue to use the students' ears (and mouths) as part of the instrument suite. Concepts such as spectrum, frequency response, distortion, quantization, and aliasing are all illustrated aurally as well as visually.

The capstone laboratory for the first course is a full-duplex optical telephone system. Each lab group constructs and tests their system individually, then must demonstrate it by communicating with another group across the length of the lab. Parameters are chosen so that the signal-to-noise ratio for the base system will be marginal and students are given suggestions for improvements they can make.

In the second course, the notion of dealing with phenomena the students can experience directly, rather than just through the instruments, is continued. Here, as befits a course featuring control, the theme is controlling "things that move." The concepts of control, as well as electronics, will be illustrated with simple electromechanical systems driven by a small motor. The capstone laboratory will be a mechanically scanned television system (expanding the theme to include "things you can see") which, as in the first course, will have to interoperate with that of another group. 


\section{COURSE EVALUATION}

The two courses were first taught during the 1997-98 academic year, which means that evaluation is, at the time of this writing, preliminary. Students were somewhat shocked the first day of class to hear how much the course was changed, but most stayed with the course. The biggest problem has been the lack of a good book; we intend to write our lecture notes and develop a text over the years.

Perhaps the most serious flaw in the course, both in terms of design and implementation, is the lack of computer engineering. At Rice, students may not have taken a programming course before taking our course. This lack hampers our ability to introduce computer organization principles. They do gain an appreciation for the flexibility inherent in software implementations, but may not appreciate the concomitant overhead.

The biggest hurdle in this course has been the students' lack of what we term "mathematical sophistication." In general, students enter Rice with strong mathematical backgrounds in that they can perform isolated calculations correctly: differentiate and integrate functions, for example. However, when it comes to generalizations of techniques they know well, uncertainty about "what to do next" quickly develops. For example, every student would know how to perform $\frac{d}{d x}(f(x)+g(x))$. However, if we ask for $\frac{d}{d x} \sum f_{n}(t)$, students generally become uncomfortable. In addition, calculations and formulas where complex numbers replace more familiar real-valued ones makes them ill at ease. Our approach is to develop engineering and mathematical ideas in parallel, taking time to make sure the mathematical manipulations are grasped.

We also use what we call for lack of a better term recursive teaching. We introduce a concept superficially at first, then come back to it over and over, each time with more sophistication and insight. Good examples of this approach are communication (the block diagram of the fundamental model is presented the first day), the complex exponential (again exhibited the first day as an example signal and re-explored with each new variant of the Fourier transform), and filtering (first arising as an interpretation of circuitbased transfer functions, then later as ideal filters for sampling and noise reduction). We find that students are initially uneasy with what they readily perceive to be a superficial grasp of the topic. We assure them that we will return to the topic, and once we do, it is our (admittedly subjective) perception that the students are grasping (albeit sometimes tenuously) many of the "advanced" concepts being presented to them. Some topics, such as error-correcting codes, have not been previously taught to undergraduate students. Student response during a recent lecture on this topic indicated insight and understanding of the technology. One area where we can definitely claim success in stimulating the students is the lab. Several students have remarked during several of the labs that "This lab is fun."

Beginning students now have a good idea what electrical engineers do; whether this knowledge attracts them to the field or not remains to be answered. Upper-level courses are now being rethought given the rather dramatic change in what entering juniors will know. The department recently voted to remove the junior-level transistor circuits course from the core curriculum (it will still be taught and can satisfy upper-level requirements) as part of the de-emphasis of discrete-element circuits as essential knowledge for electrical engineers. Clearly, the Rice electrical engineering curriculum is changing much more dramatically than in the past. These changes do have well-defined goals and moti- vation, with repackaging (re-ordering what we currently teach or teaching it differently) not the highest priority. It is our view that such changes should occur much more frequently as we work with alumni, industrial recruiters, and graduate schools to assess the quality of our undergraduate program. 
